\title{
Activated microglia-induced neuroinflammatory cytokines lead to photoreceptor apoptosis in age- related macular degeneration-like mice model
}

\section{Jing Wu}

Tenth People's Hospital of Tongji University https://orcid.org/0000-0002-7829-944X

Ge Gao

Tongji University

Fanjun Shi

Second Affiliated Hospital of Soochow University

Chaoyang Zhang

Tongji University

\section{Hai Xie}

Tongji University

\section{Qian Yang}

Tongji University

\section{Dandan Liu}

Tongji University

\section{Sichang Qu}

Tongji University

Haifeng Qin

Changhai Hospital

\section{Guo-Tong Xu}

Tongji University

\section{Fang Liu}

Shanghai Tenth People's Hospital of Tongji University

Jingfa Zhang ( $D$ 13917311571@139.com )

https://orcid.org/0000-0003-0601-4342

\section{Research}

Keywords: Age-related macular degeneration, Microglia, Photoreceptor, Neuroinflammatory cytokines

Posted Date: May 13th, 2020

DOl: https://doi.org/10.21203/rs.3.rs-27465/v1 
License: (c) (i) This work is licensed under a Creative Commons Attribution 4.0 International License. Read Full License 


\section{Abstract \\ Background}

Age-related macular degeneration (AMD) is mainly characterized by progressive deposits of drusen and photoreceptor apoptosis. Due to amyloid $\beta(A \beta)$ is the main component of drusen, there is a great possibility that $A \beta$-induced activated microglia leads to inflammation, and plays a critical role in the pathogenesis of AMD. However, the relationship between activated microglia-mediated neuroinflammatory cytokines and photoreceptor apoptosis still remains unclarified.

\section{Results}

In this study, we demonstrated that subretinal injection of $A \beta_{1-42}$ induced the microglia activation and increased inflammatory cytokines, gave rise to photoreceptor apoptosis in mice. Our results were verified in vitro by co-culture of $A \beta_{1-42}$ activated primary microglia and photoreceptor cell line $661 \mathrm{~W}$, and we also performed that p38 MAPK signaling pathway was involved in $A \beta_{1-42}$ induced microglia activation and inflammatory cytokines release.

\section{Conclusions}

Overall, our findings indicated that activated microglia-mediated neuroinflammatory cytokines contributed to photoreceptor apoptosis under the stimulation of $A \beta_{1-42}$. Moreover, this study will provide a potential preventive and therapeutic approach for AMD treatment.

\section{Background}

Age-related macular degeneration (AMD) is the most common and progressive retinal degenerative disease, with the prevalence increased exponentially with aging[1-4]. According to the retinal pathologies, AMD is divided into "dry" and "wet" forms, with the majority is dry form, characterized by progressive deposits of drusen and photoreceptor apoptosis. Amyloid $\beta(A \beta)$, a peptide of 39 to 43 amino acids and the major constituent of drusen in retina and senile plaque in brain, is thought to initiate microglia activation $[5,6]$ and neurotoxic events in patients with AMD and Alzheimer's Disease (AD) [710]. In amyloid precursor protein (APP) transgenic mice model, $A \beta$ deposition accumulates between retinal pigment epithelium (RPE) and Bruch's membrane, which is associated with microglia activation and RPE dysfunction[11]. Atrophy or loss of photoreceptor is another important pathological feature in AMD, leading to the severe visual loss $[12,13]$. However, the link between microglia activation and photoreceptor degeneration has not be fully elucidated yet.

Inflammation response caused by microglia plays a critical role in the pathogenesis of $\operatorname{AMD}[14,15]$. Microglial cells are the major resident immune cells in the central nervous system (CNS) and the retina, 
displaying a few of distinctive features and profoundly being influenced by the local microenvironment[16]. Under physiological conditions in retina, microglia are usually restricted in the inner retina, including the nerve fiber layer (NFL), ganglion cell layer (GCL), inner plexiform layer (IPL) and inner nuclear layer (INL), with a ramified morphology. Microglia could be activated once the retinal homeostasis was disturbed and migrate from the inner retina to the outer retina, including the outer nuclear layer (ONL), and even the subretinal space, with the characteristic amoeboid morphology, further releasing inflammatory cytokines to facilitate pathological destruction[17]. As a result, $A \beta$ deposition in the retinas of patients with AMD may activate microglia and provoke its production of proinflammatory cytokines, which ultimately results in microglial-mediated neuroinflammation and retinal degeneration.

Photoreceptor degeneration is a remarkable pathological feature during AMD processing, while cellautonomous signaling within photoreceptors are vital to AMD. However, few studies addressed the noncell-autonomous manner between microglia-derived neuroinflammatory cytokine and photoreceptor death in the pathogenesis of AMD. In dry AMD, the activated microglia migrate to the $\mathrm{ONL}$, where the cell body of photoreceptor localizes. Therefore, we wondered whether the non-cell-autonomous mechanism could mediate photoreceptor cell apoptosis by activated microglia.

In this study, we mimicked an inflammatory milieu in $\mathrm{C} 57 \mathrm{bl} / 6 \mathrm{~J}$ mouse by subretinal injection of $A \beta_{1-42}$ and studied the relationship between microglia activation and photoreceptor apoptosis in retina. The effects were detected by using $A \beta_{1-42}$ treated primary microglial cells on photoreceptor cell line (661W). Meanwhile, the p38 mitogen-activated protein kinase (MAPK) pathway mediated neuroinflammatory cytokines release was also explored in microglia. Therefore, by modulating neuroinflammatory cytokines produced by microglial activation it represents potential as a possible preventive and therapeutic approach for AMD.

\section{Results}

\section{Retinal functional deterioration and photoreceptor degeneration after subretinal injection of $A \beta_{1-42}$ in mice}

To testify whether visual function was impaired by $A \beta_{1-42}$, ERG was performed in mice on day 1 , day 3 and day 7 after subretinal injection of $A \beta_{1-42}$. Compared with those in PBS-treated mice, the scotopic awave and b-wave ERG amplitudes were decreased in a time-dependent manner in $A \beta_{1-42}$ injected groups. The average amplitude of a-wave was decreased by $15.8 \%$ on day $1,61.4 \%$ on day 3 , and $67.0 \%$ on day 7 ; and the average amplitude of b-wave was decreased by $20.4 \%$ on day $1,51.4 \%$ on day 3 , and $64.1 \%$ on day 7, respectively (Fig. 1A). The lower in ERG wave amplitudes generally implies the lasting deterioration in photoreceptor and RPE.

To detect the apoptosis of photoreceptor, TUNEL assay and the thickness of ONL measurement were performed in mice after subretinal injection of $A \beta_{1-42}$ on day 1 , day 3 and day 7 . One day after subretinal 
injection of $A \beta_{1-42}$, isolated TUNEL-positive photoreceptors were detected in the ONL, there was no difference between $A \beta_{1-42}$ induced mice and PBS-injected control mice (Fig. 1B). At day 3 , the amounts of photoreceptor apoptotic nuclei significantly increased in the ONL,and was about 4.5-fold of that than in control. At day 7, the number of photoreceptor apoptotic nuclei was fallen down to relatively normal level. The thickness of ONL was decreased by time during this process, and it was obviously decreased to about $70.1 \%$ on day 3 (Fig. 1B).

To further confirm the apoptosis after subretinal of $A \beta_{1-42}$ in mice, we performed Western blot to examine the protein levels for early indicators of apoptosis, including the total and cleaved caspase 3 (a substrate of activated caspase-3) protein expressions on day 3 , and the data showed both total and cleaved caspase 3 protein levels were increased significantly in retina in $A \beta_{1-42}$ injected mice compared with that in control, i.e., about 1.9-and 1.6-fold for total and cleaved caspase 3 (Fig. 1C). Altogether, these data suggest that the subretinal injection of $A \beta 1-42$ could induce to visual function deterioration and photoreceptor apoptosis aggravation, which led to AMD-like pathology in mice.

\section{$A \beta_{1-42}$ induced microglia activation and pro-inflammatory cytokines expression in mice}

To access whether $A \beta_{1-42}$ deposition could induce microglia activation after subretinal injection, we performed microglia immunostaining (Iba-1 positive) on both retinal cryosections and flat-mount. Compared with control, the expression of Iba-1positive cells was elevated abundantly at day 3 after subretinal injection. However, the activation of retinal microglia was down regulated in $A \beta_{1-42}$ induced AMD mice at day 7. As shown in Fig. $2 A$, from retinal cryosections, the amount of microglia was increased significantly on day 3. Importantly, while lba 1 positive cells were barely found in the outer retina in control mice, we could observe the distribution of microglia population partly migrated from the inner retina towards the $O N L$ and subretinal space after subretinal injection of $A \beta_{1-42}$ on day 3 (Fig. 2A). The results were confirmed on retinal flat-mounts (Fig. 2B), in the control mice, most of the Iba-1 positive cells had a ramified appearance and their processes were retracted through the retina. While in $A \beta_{1-42}$ induced mice, the appearance of Iba-1 positive cells were converted to amoeboid type with morphological features, including: with larger bodies (about 3.2 fold) and extension of processes across the ONL to intercalate to photoreceptor somata. (Fig. 2B-C).

To further characterize the activation of microglia, we detected the TSPO, CD86 and inflammatory factor in the retinas of $A \beta_{1-42}$ induced $A M D$ model mice compared with control group on day 3 using Western blot. The protein levels of TSPO and CD86 were dramatically higher in the retina of $A \beta_{1-42}$ induced AMD mice, which were about 1.9- and 1.5-fold of that in the control (Fig. 2D), indicating the activation of microglia. Besides, the pro-inflammatory factors such as IL-1 $\beta$ and cyclooxygenase-2 (COX-2) were also detected, since these factors were reported to be produced by activated microglia. Compared with the controls, $A \beta 1-42$ treated group resulted in significant increases in the IL-1 $\beta$ and COX-2 expressions on day 3, i.e., 1.5- and 2.5-fold of the control (Fig. 2D). The data showed that subretinal injection of $A \beta_{1-42}$ 
induced microglia activation and migration from inner to outer retina, accompanying with increased proinflammatory cytokines expression and photoreceptor apoptosis on day 3 .

\section{$A \beta_{1-42}$ induced primary microglial activation and pro-inflammatory cytokine expression in vitro}

To further investigate whether $A \beta_{1-42}$ was the activator of microglia and modulated pro-inflammatory cytokines secretion, primary microglia cells were employed and treated with $A \beta_{1-42}$ in vitro. First, to test the cellular compatibility of the primary microglia treated with different concentrations $(0.5 \mu \mathrm{mol} / \mathrm{L}$ to $10 \mu \mathrm{mol} / \mathrm{L}$ ) of $A \beta 1_{-}{ }_{42}$, we monitored the viability of the cultured primary microglia using the CCK-8 assay. The cell viability was dose-dependently decreased by $A \beta_{1-42}$, when the concentration of $A \beta_{1-42}$ reached $4 \mu \mathrm{mol} / \mathrm{L}$, the number of viable cells declined significantly, which was about $59.7 \%$ of the control (Fig. 3A). Based on this result, $2 \mu \mathrm{mol} / \mathrm{L} \mathrm{A} \beta_{1-42}$ was selected as the optimal concentration in our subsequent experiments. The morphology of microglia displayed differently between control and $A \beta_{1-42}$ treated group. In control group, the cellular body of the basal homeostatic microglia was relatively smaller with ramified morphology after 24 hours' culture. Conversely, $A \beta_{1-42}$-treated microglial cells converted to larger cellular body with amoeboid morphology (Fig. 3B). The morphological features suggested that primary microglia may be activated to adopt an amoeboid shape by $A \beta_{1-42}$ stimulation.

To further confirm our findings, we examined the protein expressions of TSPO. TSPO protein expression was increased within 24 hours of $A \beta_{1-42}$ treatment, which was about 2.3-fold of the control. To verify the inflammatory production for $A \beta_{1-42}$ stimulation on the primary microglia, western blot analysis indicated that the levels of IL-1 $\beta$ and COX-2 were also upregulated in microglial cells for 24 hours of $A \beta_{1-42}$ treatment in vitro, i.e., 1.7- and 3.2-fold of the control (Fig. 3E-3F). All of these results suggested that microglial activation be induced by $A \beta_{1-42}$ stimulation, and activated microglia released proinflammatory cytokines in this process.

\section{The inflammatory cytokines produced by $A \beta_{1-42}$-treated primary microglial cells increased the apoptosis of $661 \mathrm{~W}$ cells}

Both in vivo and in vitro data demonstrated the activation of microglia, the increased inflammatory cytokines production as well as the apoptosis of photoreceptors in mice retina under the treatment of $A \beta_{1}$ -42 . It deserved the exploration that whether the apoptosis of photoreceptors could be induced directly by the activated microglia derived inflammatory cytokines. To do this, we collected the conditioned medium (MCM) from the supernatant of the primary microglia cell treated with $A \beta_{1-42}$, and then cultured $661 \mathrm{~W}$ with it. We detected cellular morphology and analyze the apoptosis of $661 \mathrm{~W}$ cells after 24 hours MCM culture, control medium was applied by the supernatant of microglial cells without adding to $A \beta_{1-42}$. Given the direct impact of $A \beta_{1-42}$ on $661 \mathrm{~W}$ culture, we also tested whether $661 \mathrm{~W}$ apoptosis was changed in basal medium (BM) adding to $2 \mu \mathrm{mol} / \mathrm{L} \mathrm{A} \beta_{1-42}$. On light microscope, $661 \mathrm{~W}$ displayed similarappearing morphological features in both $C M$ and $B M$ with $A \beta_{1-42}$ cultured $661 \mathrm{~W}$ for 24 hours, however, the cell density of $661 \mathrm{~W}$ incubated with MCM was significantly reduced from 18 hours to 24 hours 
(Fig. 4A). Western blot analysis showed after 24 hours cultured of $661 \mathrm{~W}$, the level of caspase 3 in $661 \mathrm{~W}$ was significantly increased in MCM-treated group, compared with $C M$ group or $B M$ with $A \beta_{1-42}$ group (Fig. 4B-C). The above data indicated that photoreceptor apoptosis might be caused by the activated microglia-derived inflammatory cytokines.

\section{P38 MAPK signaling pathway was involved in microglia activation and photoreceptor apoptosis under A $\beta$ $1-42$ treatment}

Since p38 MAPK signaling pathway was one of signaling pathway involved in the process of microglia, thus, we explored whether p38 MAPK signaling pathway exerts its function in photoreceptor apoptosis via $A \beta_{1-42}$ induced microglia activation in retina. Both total and phosphorylated p38 MAPK protein levels in retina were examined on day 3 after subretinal injection in mice with $A \beta_{1-42}$ or with PBS as control. As shown in Fig. 5A, the ratio of phosphorylated to total level of p38 was accelerated greatly in $A \beta_{1-42}$ treated group, which was about 2.3-fold of that in control. This result was also confirmed in vitro and the expression of p38 MAPK was about 3.1-fold of that in the control (Fig. 5B). These effects implicated the possibility that $p 38$ MAPK signaling pathway was associated with $A \beta_{1-42}$ induced microglia activation.

Taking into account that our preliminary data revealed that p38 MAPK protein expression was elevated in $A \beta_{1-42}$ induced microglial cells both in vivo and vitro, we wondered if p38 MAPK favors its downstream the inflammatory cytokines secretion in microglia, as inflammatory cytokines initiating the apoptosis of photoreceptor. To address this issue, we used p38 MAPK inhibitor to evaluate the role of p38 MAPK on microglial cell secretion of inflammatory cytokines with Q-PCR. $10 \mu \mathrm{mol} / \mathrm{L}$ BIRB 796 was added to microglia cultures for $1 \mathrm{~h}$ before treatment with $2 \mu \mathrm{mol} / L A \beta_{1-42}$ for 24 hours. As expected, the mRNA expressions of inflammatory cytokines in $A \beta_{1-42}$ treated group, such as IL-1 $\beta, C O X-2$, TNF- $a$ and iNOS, were dramatically increased, which was about 5.1-, 6.1-, 3.2-, and 2.7-fold, respectively, of that in control. BIRB 796 largely abolished such increases and phosphorylated p38 MAPK protein level decreased in $A \beta_{1-42}$ induced microglia cultures correspondingly (Fig. 5B-C). These results are in accordance with our previous hypothesis that the p38 MAPK signaling pathway may have a vital role in $A \beta_{1-42}$ induced microglial activation and its secretion of inflammatory cytokines, all of which may play an important role in photoreceptor apoptosis.

\section{Discussion}

In this study, we demonstrate that $A \beta_{1-42}$ induces the microglia activation and inflammatory cytokines secretion both in early AMD mice model and in primary microglial cells, leading to the disfunction of retina in vivo and $661 \mathrm{~W}$ apoptosis in vitro. During these processes, the trend of photoreceptor apoptosis is consistent with the level of activated microglia and inflammatory cytokines. More importantly, we uncover that inflammatory cytokines produced by activated microglia initiate the photoreceptor apoptosis, otherwise than the direct toxic effect of $A \beta_{1-42}$, few prior studies were focused on the non-cellautonomous microenvironmental interactions between microglia and photoreceptor in AMD. Furthermore, 
we show that $\mathrm{p} 38$ MAPK signaling pathway is involved in modulating inflammatory cytokines secretion in $A \beta_{1-42}$ induced microglia, inhibition of the phosphorylated p38 MAPK effectively blocks inflammatory cytokines released by the activated microglia.

In clinic, AMD displays the accumulation of $A \beta$ in the drusen beneath the RPE, A $\beta$-targeted agents and anti-inflammatory therapies are still under evaluation for the treatment of AMD[18-20]. Accordingly, it is required to elucidate the mechanisms of $A M D$ is of great importance to understand its pathogenesis and the effective treatments. To detect the harmful effect of $A \beta_{1-42}$ in retina, we examined $E R G$ and photoreceptor apoptosis after $A \beta_{1-42}$ injection in mice model. Results showed that the amplitudes of both $a$ - and b-waves were significantly reduced, while the number of apoptotic photoreceptors nuclei was increased significantly, accompanying the thinning of $O N L$ on day 3 in $A \beta_{1-42}$ treated mice (Fig. 1). These ultrastructural alterations correspond to retinal functional changes in AMD process, as quantified by ERG declining and the progressive retinal degeneration.

Microglial activation is an important hallmark of neuroinflammatory diseases including $\operatorname{AMD}[14,21,22]$, as resident immunizing cells in retina, microglia sustain microenvironmental equilibrium and its dysfunction initiates a series of retinal degenerative performance [17, 23-26]. Therefore, considered that there was no well-established AMD animal models to mimic both the etiological and morphological properties of $\operatorname{AMD}[27,28]$, we focused on microglial activation by subretinal injection of $A \beta_{1-42}$ in mice and studied its indirect effect on photoreceptors by releasing the inflammatory cytokines. Our observations showed that $A \beta_{1-42}$ initiated microglial activation and inflammatory response after subretinal injection in mice model (Fig. 2). Activated microglia displayed the amoeboid morphology with enlarged cellular bodies and infiltrated the ONL from inner retina (Fig. 2A-B), which indicate that activated microglia may contribute to retinal neuronal dysfunction and photoreceptor apoptosis in AMD.

Since neuroinflammation is a remarkable feature involved in the early phase of AMD procession[29], both in vivo and in vitro, the alterations in neuroinflammatory cytokine expression profiles were similar (Fig. 2D, Fig. 3C-F) in our study, the increased inflammatory cytokines like IL-1 $\beta$ and COX-2 were coincided with the published ones. Combining the ultrastructure and functional deterioration in our study, the $A \beta_{1-42}$ induced subretinal injection mice model may generally be used to as an early AMD mice model in further research. To the best of our knowledge, this is a novel model to mimic the pathogenesis of AMD and study the related mechanisms both in vivo and in vitro.

As the dry AMD progresses, visual loss is generally regarded as a consequence of photoreceptor degeneration, so it is critical to explore the mechanism of photoreceptor dysfunction or death in the early onset of AMD. As we known, microglial activation plays a vital role in the etiology and development of $A M D$,it is interesting to study the underlying relationship between microglia activation and photoreceptor apoptosis. As observed in $A \beta_{1-42}$ induced mice model, activated retinal microglia infiltrated the ONL and photoreceptor cells underwent apoptosis, and the quantitative analysis demonstrated photoreceptor apoptosis in the ONL performed consistent trend with microglia activation and migration via dynamic processes, peaking on day 3 and falling to a lower level on day 7. Furthermore, it showed the increased 
apoptosis of $661 \mathrm{~W}$ cells under the treatment of MCM derived from activated microglia in vitro, while $A \beta_{1-42}$ had no direct function of inducing cell death in $661 \mathrm{~W}$ cells basal medium (Fig. 4A-C). Therefore, our study indicated that the cellular microenvironment produced by $A \beta_{1-42}$ activated microglia may have a critical function on photoreceptor apoptosis, which means $A \beta_{1-42}$ activated microglia and its related inflammatory cytokines may have a detrimental effect on photoreceptor. In other words, photoreceptor apoptosis was partly due to the increased inflammatory cytokines by activated microglia during the early onset of AMD.

It has been reported that there is an elevated phagocytic uptake of apoptotic photoreceptor fragments by activated microglia/macrophage whey acidic protein (AMWAP)-treated microglia[21], we infer that microglia may recognize and phagocytose apoptotic photoreceptor debris directly[30], however, the causal effect of neuroinflammatory cytokines released by activated microglia on photoreceptor death cannot be excluded. To prove this, activated derived MCM containing the inflammatory factors such as IL-1 $\beta, \operatorname{COX} 2$, and etc. could induce photoreceptor apoptosis in our culture system, which confirmed the non-cell-autonomous interactions between the activated microglia and photoreceptors. To further clarify the pathways mediated inflammatory cytokines release by activated microglia, the p38 MAPK signaling pathway was studied. Accumulated studies reported that p38 MAPK signaling pathway was critical in many age-related degenerative diseases[31-33], and it was also a major pathway underlying activation of microglia required in AMD[34]. By attenuating the activation of p53 and p38 in a Müller cell ablation transgenic mice model, photoreceptor degeneration was prevented obviously[35], so we tested whether p38 MAPK expression was upregulated in $A \beta_{1-42}$ induced mice in vivo. Furthermore, with BIRB 796, the inhibitor of p38 MAPK phosphorylation, we found that the mRNA levels of inflammatory cytokines, including IL-1 $\beta$, COX-2, TNF- $\alpha$ and iNOS, were decreased significantly under the treatment of BIRB 796 in microglia (Fig. 5C). Our results were consistent with previous studies[36-38] that p38 MAPK signaling was vital in activated microglia to release inflammatory cytokines, these findings also indirectly confirmed our hypothesis that activated microglial may lead to the apoptosis of photoreceptor by releasing inflammatory cytokines, which provides a plausible explanation about the mechanism between $A \beta_{1-42}$ induced microglia and photoreceptor apoptosis in AMD (Fig. 6).

Interestingly, inflammatory cytokines produced by microglial activation leads to the apoptosis of photoreceptor. Reviewing the related studies about the mechanisms of photoreceptor apoptosis in retinal degenerative diseases, RPE degeneration was the primary candidate due to its supportive functions[3941], such as secretion of growth factors, phagocytosis of residual photoreceptor outer segments, spatial buffering of ions, etc. In this study, however, we uncovered activated microglia-derived neuroinflammatory cytokines also played a detrimental role in photoreceptor apoptosis during the early stage of AMD, which might add a new mechanism of photoreceptor apoptosis in the pathogenesis of dry AMD.

\section{Conclusions}


In summary, our study indicated the feasibility of creating a nurturing environment for AMD mice model with subretinal injection of $A \beta_{1-42}$, which might be considered as a valuable research tool for the early stage of AMD. Through modulating p38 MAPK signal pathway, activated microglia can proliferate, migrate and produce neuroinflammatory cytokines towards the impaired sites to initiate tissue damage. Non-cell-autonomous microglia-photoreceptor interactions may play a detrimental role in the AMD progression, the inflammatory cytokines released by active microglia could contribute to photoreceptor apoptosis. Hopefully, our preliminary results may represent a potential preventive and therapeutic approach for AMD treatment.

\section{Methods}

\section{$A \beta_{1-42}$ oligomerization}

$A \beta_{1-42}$ oligomeric peptide (Sigma-Aldrich Company, Germany, Catalog Number A9810) was dissolved with anhydrous dimethyl sulfoxide (DMSO) to the concentration $500 \mu \mathrm{mol} / \mathrm{L}$ as previous described[42]. And then the stock solution $(100 \mu \mathrm{mol} / \mathrm{L})$ was make phosphate-buffered saline (PBS) and stored at $20^{\circ} \mathrm{C}$ before use.

\section{Animals And Subretinal Injection}

C57bl/6J mice (Slaccas Laboratory Animal, Shanghai, China) were obtained at room temperature under a 12-hour light-dark cycle. All the animal studies were conducted according to the ARVO Statement for the Use of Animals in Ophthalmic and Vision Research. The protocol was approved by the Committee on the Ethics of Animal Experiments of the tenth people's hospital affiliated Tongji University (Permit Number: SHDSYY-2020-2938). At least three individuals were performed for each experiment.

The subretinal injection was performed according to the previous studies[18]. Twelve-week-old C57bl/6J mice were anesthetized by an intraperitoneal (IP) injection of $4 \%$ chloral hydrate, and the pupils were

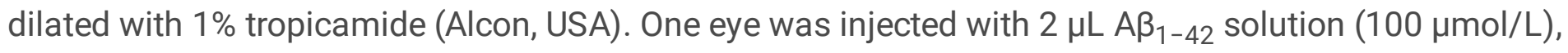
and the other eye was injected with $2 \mu \mathrm{L}$ PBS (vehicle). Briefly, a 30-gauge needle puncture the sclera 2$\mathrm{mm}$ behind the limbus to make a tunnel. Then $2 \mu \mathrm{L} A \beta_{1-42}$ solution or PBS was subretinal injected using a 33-gauge blunt needle (Hamilton Company, Reno, NV, USA).

\section{Electroretinography (ERG)}

RETIscan System (Roland Consult, Brandenburg, Germany) was used for scotopic ERG with signal stimulation, amplification and transportation. The protocol was followed as previous described [43], the ERG was carried out on day 1, day 3, and day 7 post-injection. Briefly, the mice were dark-adapted over 16 hours before performing ERG, mice were general anesthetized with an IP of $4 \%$ chloral hydrate under dim 
red illumination. Pupils of mice were dilated with $1 \%$ tropicamide for at least 20 minutes. After $0.5 \%$ Proparacaine hydrochloride (Alcon) applied for topical anesthesia of the cornea, contact lens electrodes with Gold wire loops were fixed on the surface of two corneas and the ground electrode was fixed in the tail, the parameters of scotopic ERG were measured. The amplitude of both a-wave and b-wave were recorded and analyzed using built-in software of ERG system.

\section{TUNEL Assay}

Apoptosis was detected with the kit of terminal deoxynucleotidyl transferase-mediated nick end labeling (TUNEL) according to the manufacturer instruction (YEASEN, China). Apoptotic cells were analyzed using Image $\mathrm{J}$ software.

\section{Immunofluorescence}

The cryosections of the retina and the primary microglia cells were permeabilized, blocked and incubated the primary antibody of Iba- 1 (1:500, Wako) overnight at $4{ }^{\circ} \mathrm{C}$. After washing thrice with PBS, the cryosections were incubated with Goat anti-Rabbit IgG conjugated to Alexa-568 (1:200, Invitrogen) for 1 hour at room temperature. After extensive wash, then the sections were counterstained with DAPI.

For the retinal whole-mount preparations, mice eyeballs were enucleated and fixed with $4 \%$ paraformaldehyde (PFA) for 2 hours, and then washed with PBS for three times. The retinas were isolated under dissecting microscope and were cut into four parts. Following the permeabilization and blocking step, the flat-mounts were incubated with the primary antibody of Iba-1 $\left(1: 500\right.$, Wako) overnight at $4{ }^{\circ} \mathrm{C}$. After washing with PBS, the retina flat-mounts were incubated with the secondary antibody labeled with Alexa-568 (1:200, Invitrogen) for 2 hours and counterstained with DAPI.

The immunofluorescence of the cryosections, retinal flat-mounts and the primary cells were examined with NIKON A1 + confocal microscope (NIKON, Japan). The pictures were analyzed using ImageJ.

\section{Protein Extraction And Western Blot Analysis}

The mice retina, primary microglial cells and $661 \mathrm{~W}$ cells were lysed by radio-immunoprecipitation assay (RIPA) buffer containing proteinase inhibitors (Beyotime, Shanghai, China). After denaturation, $30 \mu \mathrm{g}$ of each sample was separated by SDS-PAGE (10-15\% gradient) gel (Beyotime, Shanghai, China) and transferred to nitrocellulose membranes (Bio-Rad). The membranes were blocked by $5 \%$ skimmed milk in Tris-buffered saline with Tween-20 (TBST) (Bright Dairy \& Food Co., Ltd., Shanghai, China) for 30 minutes at room temperature. Then the membranes were incubated with the primary antibodies against TSPO (1:1,000, abcam, No. ab92291), CD86 (1:500, abcam, No. ab112490), COX2 (1:1,000, abcam, No. ab15191), IL-1ß (1:1,000, abcam, No. ab9722), caspase 3 (1:1,000, cell signaling Technology, No.9662), cleaved caspase 3 (1:1,000, cell signaling Technology, No.9661), Phospho-p38 MAPK (1:1,000, Cell 
Signaling Technology, No.4511), p38 MAPK (1:1,000, Cell Signaling Technology, No.8690) or $\beta$-actin $\left(1: 5,000\right.$, abcam) overnight at $4{ }^{\circ} \mathrm{C}$. After washed thrice with TBST, the corresponding secondary antibodies were incubated at room temperature for 1 hour, and then washed thoroughly with TBST. The membranes were imaged with Odyssey infrared imaging system (LI-COR Biosciences, Lincoln, NE). The optical density of each band was quantified by using Quantity One software (Bio-Rad), and was normalized with $\beta$-actin.

\section{Primary Microglial Cell And 661W Cell Culture}

Mouse primary microglial cells were isolated from the brains of $\mathrm{C} 57 \mathrm{bl} / 6 \mathrm{~J}$ mice at postnatal day 4 or day 5 according to previously described[44]. After detaching from brain tissues, microglial cells were maintained in $75 \mathrm{~cm}^{2}$ flasks containing DMEM supplemented with $10 \%$ fetal bovine serum (FBS, Gibco), $10 \mathrm{ng} / \mathrm{mL} \mathrm{GM}-\mathrm{CSF}, 50 \mathrm{U} / \mathrm{mL}$ penicillin and $50 \mathrm{mg} / \mathrm{mL}$ streptomycin in a $37 \circ \mathrm{C}$ incubator. The microglial cells culture medium was replaced every 3 days and was identified with Iba-1 immunostaining (1:500, WAKO). The microglia were plated at $5 \times 105$ cells $/ \mathrm{mL}$ in twelve-well plate for further study.

$661 \mathrm{~W}$, a murine photoreceptor-like cell line, was cultured in DMEM supplemented with $10 \% \mathrm{FBS}, 1 \%$ penicillin and streptomycin (Invitrogen) under $5 \% \mathrm{CO} 2$ in the incubator at $37^{\circ} \mathrm{C}$.

\section{Cell Viability Assay}

Cell counting kit-8 (CCK-8) assay (MCE, USA) was used to detect microglial cells viability according to manufacturer's instruction. Microglial cells were seeded in 96-well plates with a density of $1.0 \times 104$ cells per well. To determine the optimal dose of $A \beta_{1-42}$ on microglia, $A \beta_{1-42}$ with the final concentration of 0 , $0.5,1,2,4,8,10 \mu \mathrm{mol} / \mathrm{L}$ was added into each well and cultured at $37^{\circ} \mathrm{C}$ for 24 hours. After that, $10 \mu \mathrm{L}$ CCK-8 reagent was added to each well. The microglial cells were incubated for 2 hours, and the absorbance value of each well was measured at $450 \mathrm{~nm}$. According to the standard curve, the corresponding cell viability was calculated.

\section{Microglial Conditioned Medium (MCM)}

Mouse primary microglial cells were cultured with $2 \mu \mathrm{mol} / L A \beta_{1-42}$ for 24 hours, then the supernatant was collected as MCM, centrifuged to remove cellular debris (5 minutes, $3,000 \mathrm{~g}$ ), MCM was applied to $661 \mathrm{~W}$ medium, then $661 \mathrm{~W}$ were plated at $3 \times 105 \mathrm{cells} / \mathrm{mL}$ in twelve-well plate for 24 hours for Western blot detection. The supernatant of primary microglial cells without adding to $A \beta_{1-42}$ was served as control medium (CM).

\section{Protein Kinase Inhibitor For P38 MAPK}


The protein kinase inhibitor for p38 MAPK (BIRB 796) was obtained from Selleck Co. (USA), dissolved in DMSO and diluted with DMEM to a concentration of $10 \mu \mathrm{mol} / \mathrm{L}$. Microglial cells were pretreated with inhibitor $(10 \mu \mathrm{mol} / \mathrm{L}$ BIRB 796) 1 hour before with $2 \mu \mathrm{mol} / \mathrm{L}$ Aß1-42 treatment in medium.

\section{Reverse-transcription Quantitative Polymerase Chain Reaction (RT-qPCR)}

Total RNA of primary microglia was isolated with RNAiso reagent (Yeasen Biotech, Shanghai, China) according to the manufacturer's instruction. Reverse transcribed using a PrimeScript ${ }^{\circledR}$ RT Reagent Kit (Yeasen Biotech, Shanghai, China). Quantitative PCR was performed with a CFX96 Real-Time PCR System (Bio-Rad, Hercules, USA) with the primers information provided in Table 1. Relative changes in gene expression were calculated using the $2^{-\Delta \Delta C t}$ method.

Table 1

पThe primer information used in this study.

\begin{tabular}{|llll|}
\hline Gene & Forward Primer (5'-3') & Reverse Primer (5'-3') & Product size (bp) \\
\hline IL-1 $\beta$ & TGCCACCTTTTGACAGTGATG & TGATGTGCTGCTGCGAGATT & 138 \\
\hline COX-2 & CATCCCCTTCCTGCGAAGTT & CATGGGAGTTGGGCAGTCAT & 178 \\
\hline TNF-a & TGTGCTCAGAGCTTTCAACAA & CTTGATGGTGGTGCATGAGA & 88 \\
\hline iNOS & CCCTTCAATGGTTGGTACATGG & ACATTGATCTCCGTGACAGCC & 158 \\
\hline -actin & AGGCGACAGCAGTTGGTTGGA & TTGGGAGGGTGAGGGACTTCCT & 166 \\
\hline
\end{tabular}

\section{Statistical analysis}

Statistical analyses were performed with OriginPro 2018 Software. All experiments were repeated at least three times. Data were expressed as mean \pm SE. Differences between groups were compared with Student t-test and one-way ANOVA, Bonferroni's test was analyzed for post hoc comparisons. A $P$-value $<0.05$ was considered to be statistically significant.

\section{Abbreviations}

$A D$

Alzheimer's disease

AMD

age-related macular degeneration

AMWAP

activated microglia/macrophage whey acidic protein

APP 
amyloid precursor protein

$A \beta$

amyloid $\beta$

CCK-8

cell counting kit-8

$\mathrm{CM}$

control medium

CNS

central nervous system

cox-2

cyclooxygenase-2

DMSO

dimethyl sulfoxide

ERG

electroretinography

FBS

fetal bovine serum

GCL

ganglion cell layer

IL-1 $\beta$

interleukin-1 $\beta$

INL

inner nuclear layer

iNOS

inducible nitric oxide synthase.

IP

intraperitoneal

IPL

inner plexiform layer

MAPK

mitogen-activated protein kinase

MCM

microglial conditioned medium

NFL

nerve fiber layer

ONL

outer nuclear layer

PBS

phosphate-buffered saline

PFA 
paraformaldehyde

RIPA

radio-immunoprecipitation assay

RPE

retinal pigment epithelium

RT-qPCR

reverse-transcription quantitative polymerase chain reaction

TBST

Tris-buffered saline with Tween-20

TNF-a

tumor necrosis factor- $a$

TUNEL

terminal deoxynucleotidyl transferase-mediated nick end labeling

\section{Declarations}

\section{Acknowledgements}

We are grateful to Binxin Wu (Tongji University School of Medicine, Shanghai, China) for technical assistance.

\section{Funding}

This work was supported by National Natural Science Foundation of China (81570852) and by Clinical Research and Cultivation Project of Shanghai Municipal Hospital, China (SHDC12019X30)

\section{Author information}

\section{Affiliations}

${ }^{1}$ Department of Ophthalmology, Shanghai Tenth People's Hospital, Tongji University School of Medicine, Shanghai, China

Jing Wu, Sichang Qu, Fang Liu

${ }^{2}$ Center for Translational Neurodegeneration and Regenerative Therapy, Shanghai Tenth People's Hospital, Tongji University School of Medicine, Shanghai, China

Ge Gao

${ }^{3}$ Department of Ophthalmology, the Second Affiliated Hospital of Soochow University, Suzhou, China Fanjun Shi 
${ }^{4}$ Department of Ophthalmology, Shanghai Tenth People's Hospital, Tongji Eye Institute, Department of Regenerative Medicine, and Department of Pharmacology, Tongji University School of Medicine, Shanghai, China

Chaoyang Zhang, Hai Xie, Qian Yang, Dandan Liu, Jingfa Zhang

${ }^{5}$ Department of Ophthalmology, Shanghai Changhai hospital, Shanghai, China

Haifeng Qin

${ }^{6}$ Department of Ophthalmology, Shanghai General Hospital (Shanghai First People's Hospital), Shanghai Jiao Tong University, Shanghai, China

Jingfa Zhang

${ }^{7}$ National Clinical Research Center for Eye Diseases; Shanghai Key Laboratory of Ocular Fundus Diseases; Shanghai Engineering Center for Visual Science and Photomedicine; Shanghai Engineering Center for Precise Diagnosis and Treatment of Eye Diseases; Shanghai, China.

Jingfa Zhang

\section{Contributions}

Jingfa Zhang and Fang Liu conceived and designed the experiments, discussed the data and revised the manuscript. Jing Wu performed most of the experiments. Fanjun Shi, Qian Yang, Dandan Liu, Sichang Qu and Haifeng Qin performed some of the experiments, analyzed and discussed the data. Ge Gao and Fanjun Shi provided the samples. Jing Wu wrote the manuscript and discussed extensively with Ge Gao, Chaoyang Zhang and Hai Xie. All authors read and approved the final manuscript.

\section{Corresponding authors}

Correspondence to Jingfa Zhang and Fang Liu

\section{Ethics declarations}

All procedures involving the animal studies were conducted in accordance with the guidelines of the Committee on the Ethics of Animal Experiments of the tenth people's hospital affiliated Tongji University (Permit Number: SHDSYY-2020-2938) and adhered to the principles of the ARVO Statement for the Use of Animals in Ophthalmic and Vision Research.

\section{Consent for publication}

Not applicable.

\section{Competing interests}


The authors declare that they have no competing financial interests.

\section{References}

1. Wong WL, Su X, Li X, Cheung CMG, Klein R, Cheng C-Y, et al. Global prevalence of age-related macular degeneration and disease burden projection for 2020 and 2040: a systematic review and metaanalysis. The Lancet Global Health. 2014;2(2):e106-e16.

2. Sivak JM. The aging eye: common degenerative mechanisms between the Alzheimer's brain and retinal disease. Invest Ophthalmol Vis Sci. 2013;54(1):871-80.

3. Wu J, Zhang X, Azhati G, Li T, Xu G, Liu F. Retinal microvascular attenuation in mental cognitive impairment and Alzheimer's disease by optical coherence tomography angiography. Acta ophthalmologica. 2020.

4. Qu SC, Xu D, Li TT, Zhang JF, Liu F. iTRAQ-based proteomics analysis of aqueous humor in patients with dry age-related macular degeneration. Int J Ophthalmol. 2019;12(11):1758-66.

5. Ohno-Matsui K. Parallel findings in age-related macular degeneration and Alzheimer's disease. Progress in Retinal Eye Research. 2011;30(4):217-38.

6. Combadière C, Feumi C, Raoul W, Keller N, Rodéro M, Pézard A, et al. CX3CR1-dependent subretinal microglia cell accumulation is associated with cardinal features of age-related macular degeneration. Journal of Clinical Investigation. 2007;117(10):2920-8.

7. Zhu M, Wang X, Schultzberg M, Hjorth E. Differential Regulation of Resolution in Inflammation induced by Amyloid- $\beta 42$ and Lipopolysaccharides in Human Microglia. J Alzheimers Dis. 2014;43(4):1237-50.

8. Cai Z, Hussain MD, Yan LJ. Microglia, neuroinflammation, and beta-amyloid protein in Alzheimer's disease. Int J Neurosci. 2014;124(5):307-21.

9. Felsky D, Roostaei T, Nho K, Risacher SL, Bradshaw EM, Petyuk V, et al. Neuropathological correlates and genetic architecture of microglial activation in elderly human brain. Nature Communications. 2019;10(1).

10. Lu Y, Guo Z, Zhang Y, Li C, Zhang Y, Guo Q, et al. Microenvironment Remodeling Micelles for Alzheimer's Disease Therapy by Early Modulation of Activated Microglia. Advanced science. 2019;6(4):1801586.

11. Dong ZZ, Li J, Gan YF, Sun XR, Leng YX, Ge J. Amyloid beta deposition related retinal pigment epithelium cell impairment and subretinal microglia activation in aged APPswePS1 transgenic mice. Int J Ophthalmol. 2018;11(5):747-55.

12. Puertas-Neyra K, Usategui-Martin R, Coco RM, Fernandez-Bueno I. Intravitreal stem cell paracrine properties as a potential neuroprotective therapy for retinal photoreceptor neurodegenerative diseases. Neural regeneration research. 2020;15(9):1631-8.

13. Gasparini SJ, Llonch S, Borsch O, Ader M. Transplantation of photoreceptors into the degenerative retina: Current state and future perspectives. Prog Retin Eye Res. 2019;69:1-37. 
14. Nebel C, Aslanidis A, Rashid K, Langmann T. Activated microglia trigger inflammasome activation and lysosomal destabilization in human RPE cells. Biochem Biophys Res Commun. 2017;484(3):681-6.

15. Grimaldi A, Pediconi N, Oieni F, Pizzarelli R, Rosito M, Giubettini M, et al. Neuroinflammatory Processes, A1 Astrocyte Activation and Protein Aggregation in the Retina of Alzheimer's Disease Patients, Possible Biomarkers for Early Diagnosis. Front Neurosci. 2019;13:925.

16. Perry VH, Nicoll JAR, Holmes C. Microglia in neurodegenerative disease. Nature Reviews Neurology. 2010;6(4):193-201.

17. Karlstetter M, Scholz R, Rutar M, Wong WT, Provis JM, Langmann T. Retinal microglia: just bystander or target for therapy? Prog Retin Eye Res. 2015;45:30-57.

18. Do KV, Kautzmann MI, Jun B, Gordon WC, Nshimiyimana R, Yang R, et al. Elovanoids counteract oligomeric beta-amyloid-induced gene expression and protect photoreceptors. Proc Natl Acad Sci U S A. 2019;116(48):24317-25.

19. Muraleva NA, Kozhevnikova OS, Fursova AZ, Kolosova NG. Suppression of AMD-Like Pathology by Mitochondria-Targeted Antioxidant SkQ1 Is Associated with a Decrease in the Accumulation of Amyloid $\beta$ and in mTOR Activity. Antioxidants. 2019;8(6).

20. Parsons CG, Ruitenberg M, Freitag CE, Sroka-Saidi K, Russ H, Rammes G. MRZ-99030 - A novel modulator of $A \beta$ aggregation: I - Mechanism of action (MoA) underlying the potential neuroprotective treatment of Alzheimer's disease, glaucoma and age-related macular degeneration (AMD). Neuropharmacology. 2015;92:158-69.

21. Aslanidis A, Karlstetter M, Scholz R, Fauser S, Neumann H, Fried C, et al. Activated microglia/macrophage whey acidic protein (AMWAP) inhibits NFkappaB signaling and induces a neuroprotective phenotype in microglia. J Neuroinflammation. 2015;12:77.

22. Ma W, Silverman SM, Zhao L, Villasmil R, Campos MM, Amaral J, et al. Absence of TGF $\beta$ signaling in retinal microglia induces retinal degeneration and exacerbates choroidal neovascularization. eLife. 2019;8.

23. Grimaldi A, Brighi C, Peruzzi G, Ragozzino D, Bonanni V, Limatola C, et al. Inflammation, neurodegeneration and protein aggregation in the retina as ocular biomarkers for Alzheimer's disease in the 3xTg-AD mouse model. Cell Death \& Disease. 2018;9(6).

24. Haenseler W, Rajendran L. Concise Review: Modeling Neurodegenerative Diseases with Human Pluripotent Stem Cell-Derived Microglia. Stem Cells. 2019;37(6):724-30.

25. He J, Zhao C, Dai J, Weng CH, Bian BSJ, Gong Y, et al. Microglia Mediate Synaptic Material Clearance at the Early Stage of Rats With Retinitis Pigmentosa. Front Immunol. 2019;10:912.

26. Kaur D, Sharma V, Deshmukh R. Activation of microglia and astrocytes: a roadway to neuroinflammation and Alzheimer's disease. Inflammopharmacology. 2019;27(4):663-77.

27. Pennesi ME, Neuringer M, Courtney RJ. Animal models of age related macular degeneration. Mol Aspects Med. 2012;33(4):487-509. 
28. Cai X, McGinnis JF. Nanoceria: a Potential Therapeutic for Dry AMD. Adv Exp Med Biol. 2016;854:111-8.

29. Arroba Al, Campos-Caro A, Aguilar-Diosdado M, Valverde ÁM. IGF-1, Inflammation and Retinal Degeneration: A Close Network. Front Aging Neurosci. 2018;10:203.

30. Joly S, Francke M, Ulbricht E, Beck S, Seeliger M, Hirrlinger P, et al. Cooperative phagocytes: resident microglia and bone marrow immigrants remove dead photoreceptors in retinal lesions. Am J Pathol. 2009;174(6):2310-23.

31. Freund A, Patil CK, Campisi J. p38MAPK is a novel DNA damage response-independent regulator of the senescence-associated secretory phenotype. EMBO J. 2011;30(8):1536-48.

32. Davis T, Brook A, Rokicki M, Bagley M, Kipling D. Evaluating the Role of p38 MAPK in the Accelerated Cell Senescence of Werner Syndrome Fibroblasts. Pharmaceuticals. 2016;9(2).

33. Chen X, Chen X, Zhang X, Wang L, Cao P, Rajamanickam V, et al. Curcuminoid B63 induces ROSmediated paraptosis-like cell death by targeting TrxR1 in gastric cells. Redox Biology. 2019;21.

34. Ma W, Cojocaru R, Gotoh N, Gieser L, Villasmil R, Cogliati T, et al. Gene expression changes in aging retinal microglia: relationship to microglial support functions and regulation of activation. Neurobiol Aging. 2013;34(10):2310-21.

35. Shen W, Lee SR, Araujo J, Chung SH, Zhu L, Gillies MC. Effect of glucocorticoids on neuronal and vascular pathology in a transgenic model of selective Muller cell ablation. Glia. 2014;62(7):1110-24.

36. Kim J-E, Park H, Choi S-H, Kong M-J, Kang T-C. Roscovitine Attenuates Microglia Activation and Monocyte Infiltration via p38 MAPK Inhibition in the Rat Frontoparietal Cortex Following Status Epilepticus. Cells. 2019;8(7).

37. Conti P, Lauritano D, Caraffa A, Gallenga CE, Kritas SK, Ronconi G, et al. Microglia and mast cells generate proinflammatory cytokines in the brain and worsen inflammatory state: Suppressor effect of IL-37. Eur J Pharmacol. 2020;875:173035.

38. Ji RR, Suter MR. p38 MAPK, microglial signaling, and neuropathic pain. Mol Pain. 2007;3:33.

39. Datta S, Cano M, Ebrahimi K, Wang L, Handa JT. The impact of oxidative stress and inflammation on RPE degeneration in non-neovascular AMD. Prog Retin Eye Res. 2017;60:201-18.

40. Esteve-Rudd J, Hazim RA, Diemer T, Paniagua AE, Volland S, Umapathy A, et al. Defective phagosome motility and degradation in cell nonautonomous RPE pathogenesis of a dominant macular degeneration. Proc Natl Acad Sci U S A. 2018;115(21):5468-73.

41. Kurihara T, Westenskow PD, Gantner ML, Usui Y, Schultz A, Bravo S, et al. Hypoxia-induced metabolic stress in retinal pigment epithelial cells is sufficient to induce photoreceptor degeneration. eLife. 2016;5.

42. Cheng YW, Chang CC, Chang TS, Li HH, Hung HC, Liu GY, et al. A 3 stimulates microglial activation through antizyme-dependent downregulation of ornithine decarboxylase. J Cell Physiol. 2018;234(6):9733-45. 
43. Fu Y, Hou B, Weng C, Liu W, Dai J, Zhao C, et al. Functional ectopic neuritogenesis by retinal rod bipolar cells is regulated by miR-125b-5p during retinal remodeling in RCS rats. Scientific Reports. 2017;7(1).

44. Gao G, Zhao S, Xia X, Li C, Li C, Ji C, et al. Glutaminase C Regulates Microglial Activation and Proinflammatory Exosome Release: Relevance to the Pathogenesis of Alzheimer's Disease. Frontiers in Cellular Neuroscience. 2019;13.

\section{Figures}


A
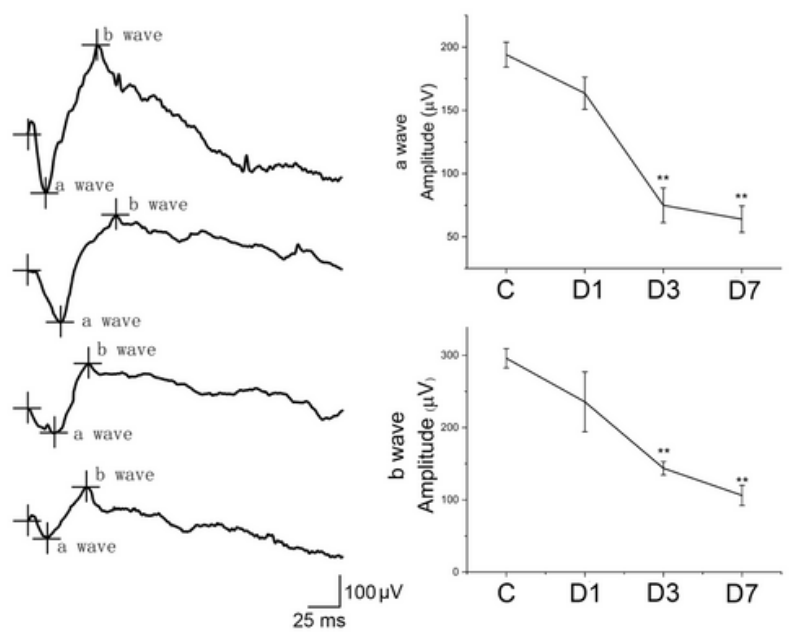

B
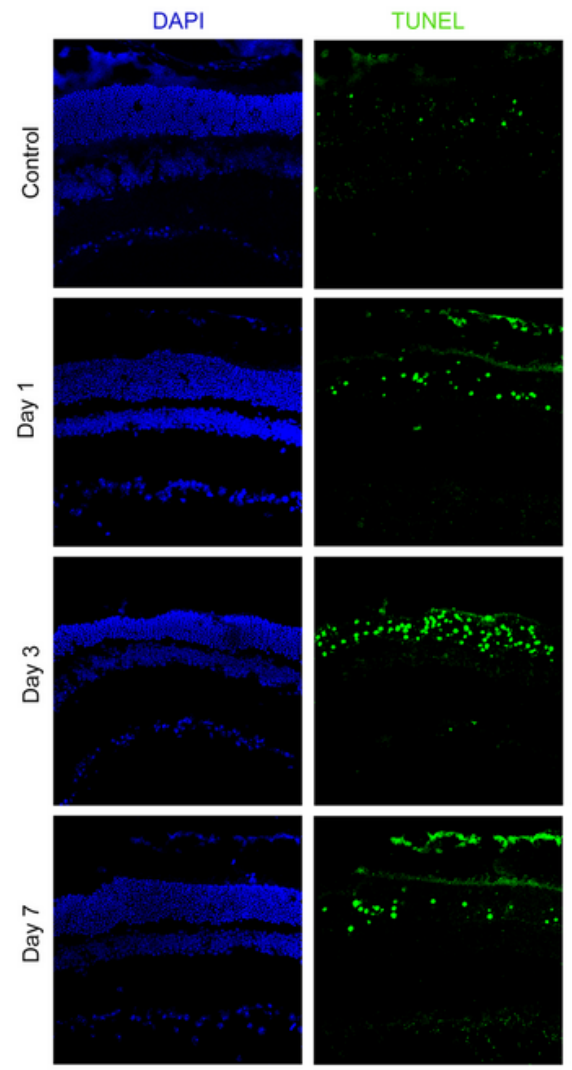

C
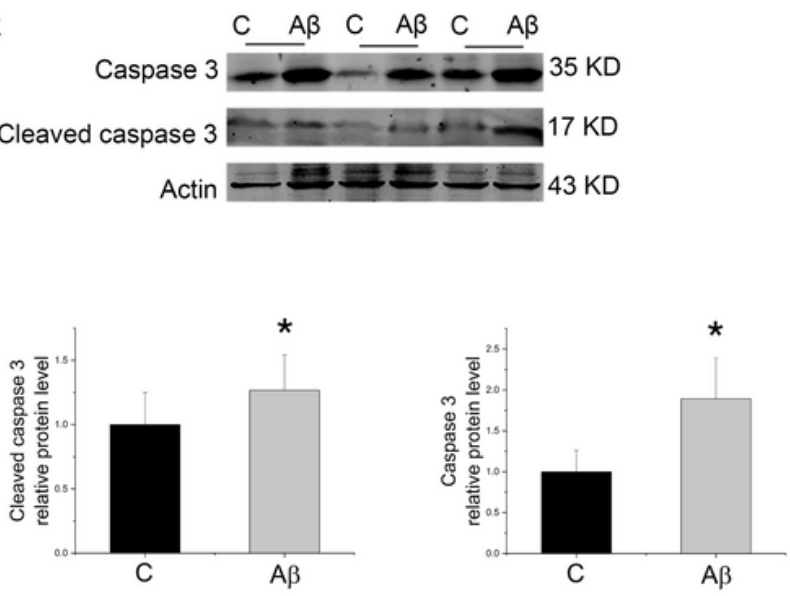

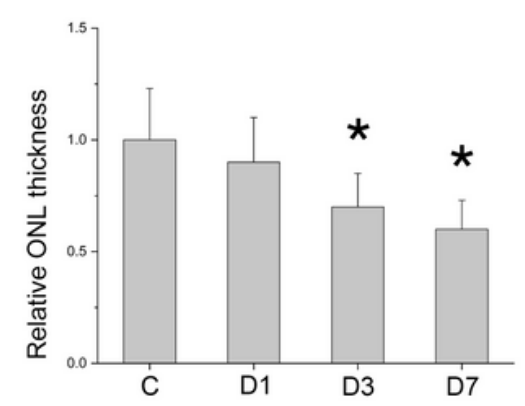

\section{Figure 1}

Retinal functional deterioration and accelerated photoreceptor apoptosis in C57BL/6J mice treated with subretinal injection of AB1-42 or PBS. (a) Representative waveforms of the maximal ERG response and the amplitudes of a-wave and b-wave of the scotopic ERG responses on day 1, 3 and 7 after treatment of A 1 1- 42. (b) Apoptosis detection with TUNEL and ONL thickness measurement in retinas with or without A 1-42treatment on day 1, 3 and 7. (c) Western blot detection of caspase 3 and cleaved caspase 3 proteins expression in mouse retina treated with $A \beta 1-42$ on day3. C, control; ONL, outer plexiform layer. 
Histograms represent the mean \pm S.E.M. $N=3, * P<0.05, \star \star P<0.01, \star \star \star P<0.001$ via Student's t-tests and one-way ANOVA, Bonferroni's test was analyzed for post hoc comparisons.

A
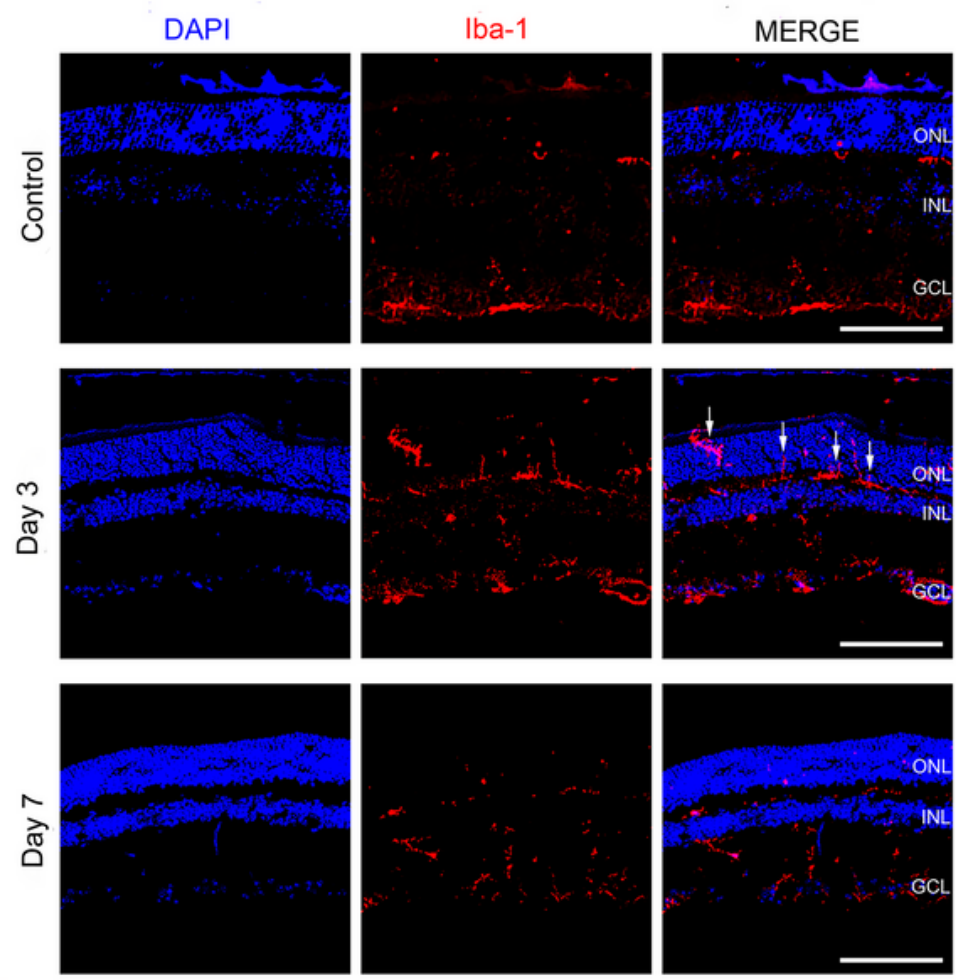

B

lba-1


C
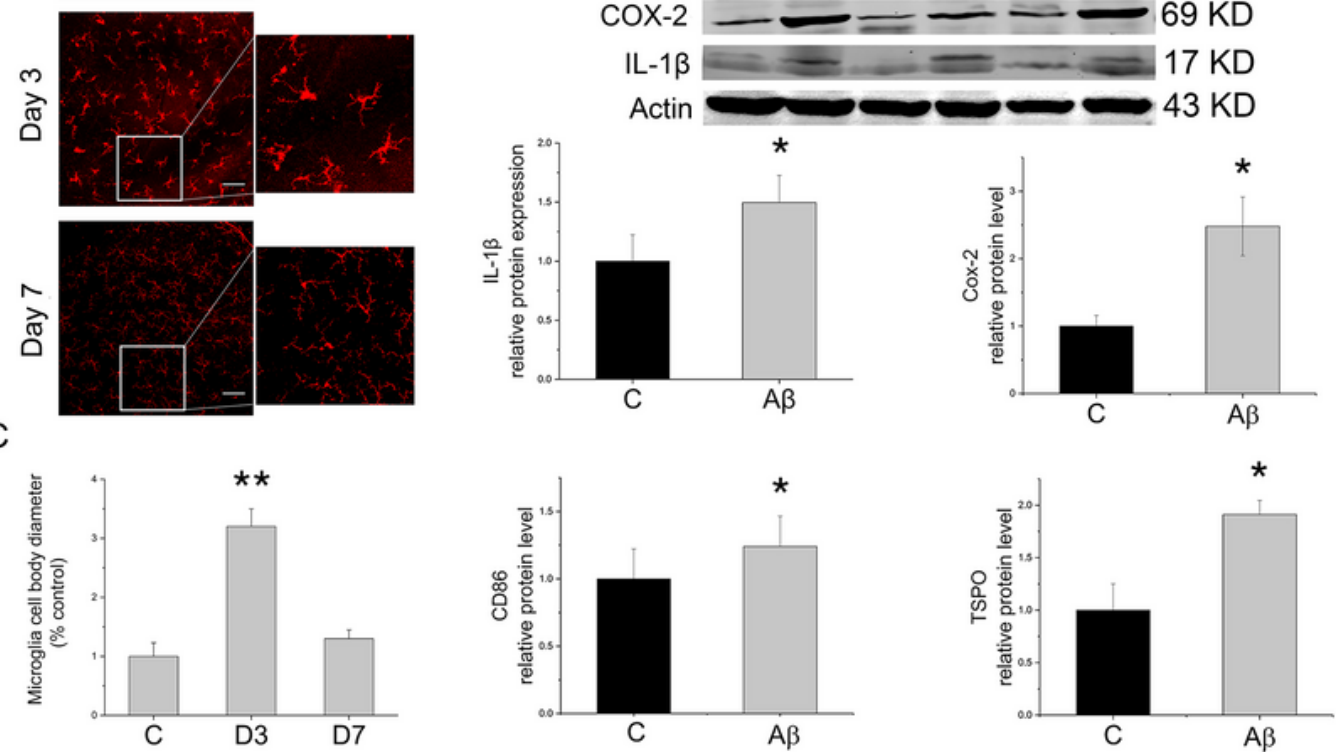

Figure 2

Microglia activation and increased inflammatory cytokines expression in C57BL/6J mice treated with subretinal injection of $A \beta 1-42$ or PBS. (A) Iba-1 immunostaining in retinas treated with $A \beta 1-42$ on day 3 and day 7 . The amount of microglia was increased significantly and migrated from inner retina to the 
outer retina on day 3. Arrows indicated the infiltration of microglia in ONL and subretinal space. (B-C) Iba1 immunostaining in retinal flat-mount on day 3 and day 7 . The morphology of lba $1+$ cells displayed the characteristic amoeboid appearance with enlarged body diameter on day 3 after treatment of AB1-42. (D) Western blot detection of CD86, TSPO, COX-2 and IL-1 $\beta$ in retina on day 3 after treatment of AB1-42. Scale bar represents $100 \mu \mathrm{m}$. GCL, ganglion cell layer; INL, inner nuclear layer; ONL, outer nuclear layer.

Histograms represent the mean \pm S.E.M. $N=3, * P<0.05$, $* \star P<0.01$, ${ }^{\star \star \star} P<0.001$ via Student's t-tests and one-way ANOVA, Bonferroni's test was analyzed for post hoc comparisons.
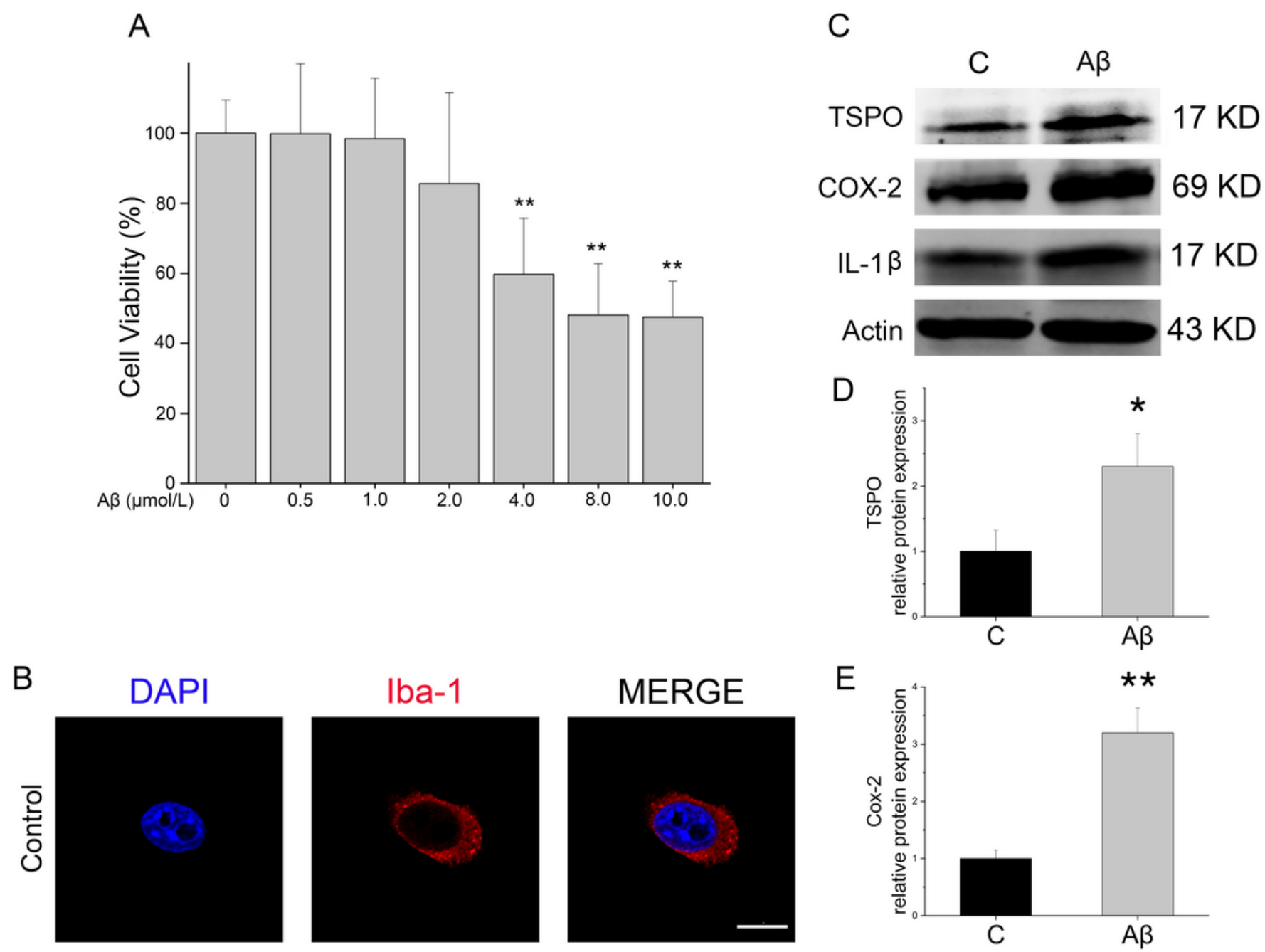

$\mathrm{E}$
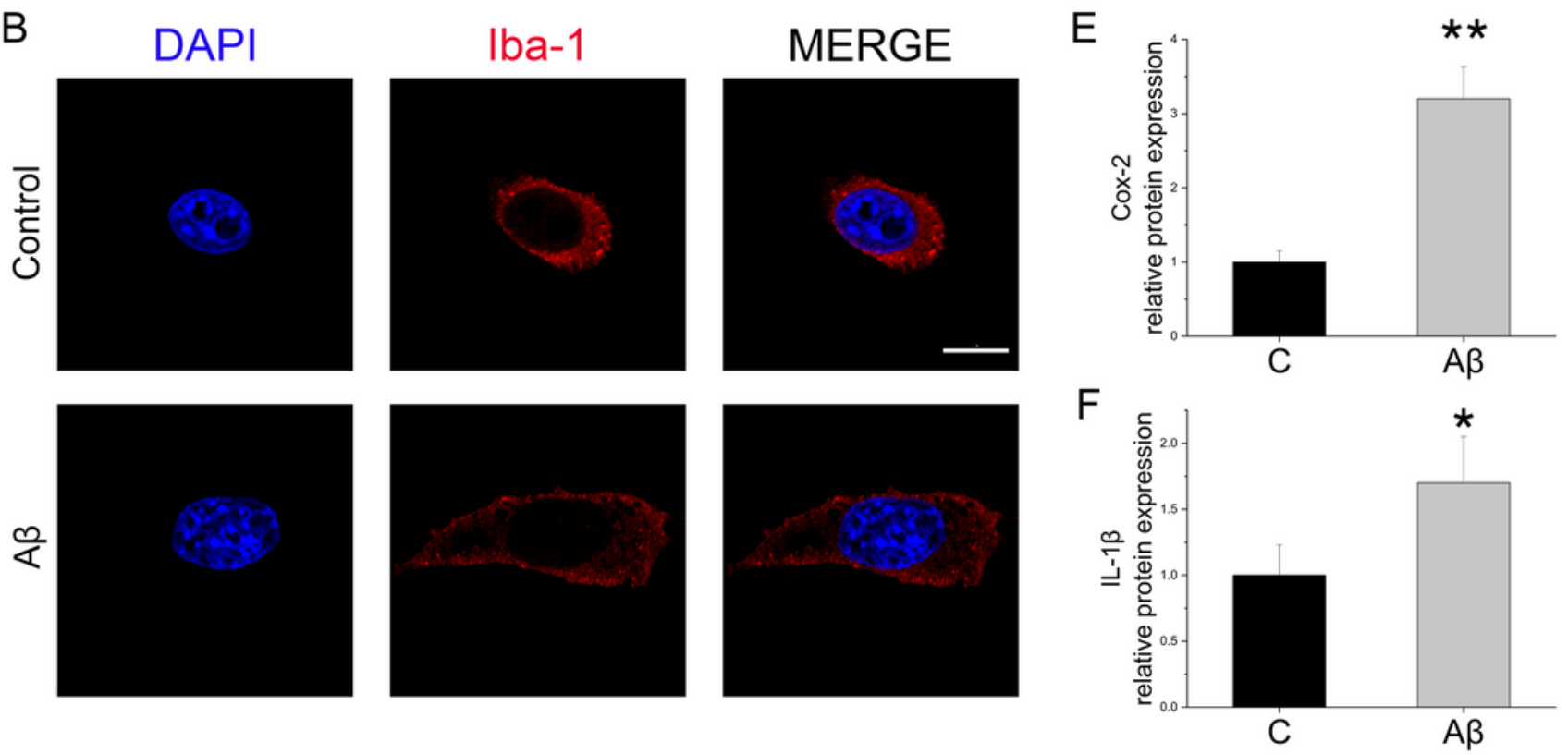

Figure 3 
A 1-42 induced primary microglial activation and pro-inflammatory cytokine expression in vitro. (A) CCK8 assay for cell viability of primary microglial cells treated with different concentrations of A 1-42 (range from $0.2 \mu \mathrm{mol} / \mathrm{L}$ to $10 \mu \mathrm{mol} / \mathrm{L}$ ) for 24 hours. (B) The representative morphology of the actived primary microglial cells with $2 \mu \mathrm{mol} / \mathrm{L} A \beta 1-42$ for 24 hours. (C-F) Western blot detection of TSPO, COX-2 and IL-1 $\beta$ in primary microglial with $2 \mu \mathrm{mol} / L \mathrm{~A} \beta 1-42$ for 24 hours. Scale bar represents $10 \mu \mathrm{m}$. Histograms represent the mean \pm S.E.M. $N=3, * P<0.05$, $* * P<0.01$, $* \star * ~ P<0.001$ via Student's t-tests and one-way ANOVA, Bonferroni's test was analyzed for post hoc comparisons.

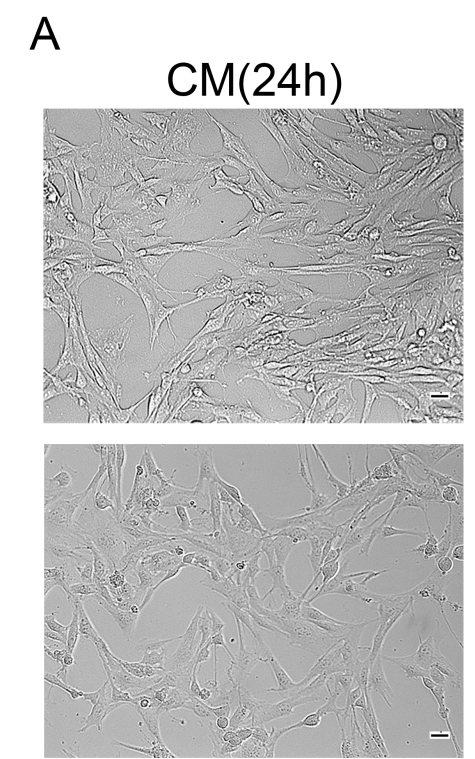

$A \beta(24 h)$
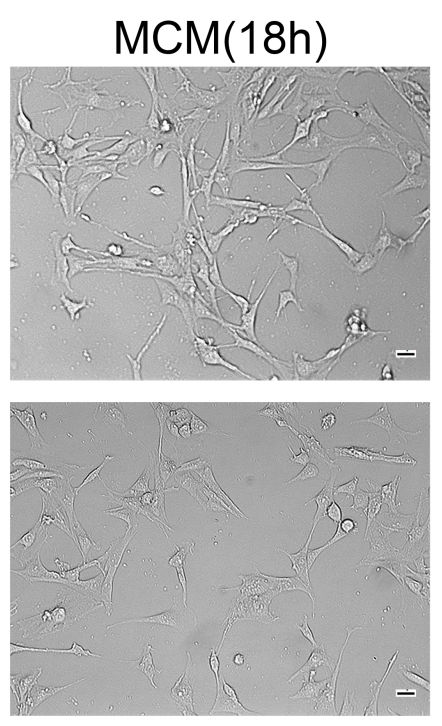

$\operatorname{MCM}(24 h)$

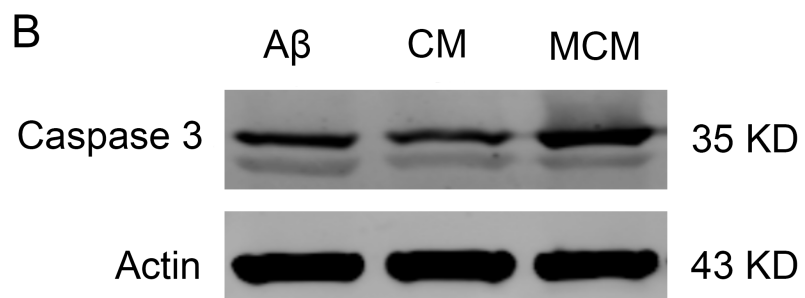

C

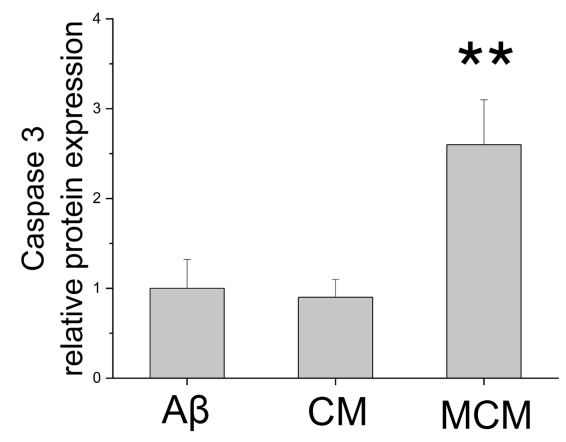

\section{Figure 4}

Activated microglia derived medium (MCM) increased the apoptosis of $661 \mathrm{~W}$ cells. (A) The representative morphology of $661 \mathrm{~W}$ treated with MCM derived from Aß1-42 treated microglia for 18 or 24 hours, CM for 24 hours, or basal medium for 24 hours, respectively. (B-C) Western blot detection of caspase 3 protein expression in $661 \mathrm{~W}$ treated with MCM, CM, or basal medium for 24 hours, respectively. CM, control medium; MCM, microglial condition medium; $A \beta$, basal medium with $2 \mu \mathrm{mol} / L A \beta 1-42$. Scale bar represents $10 \mu \mathrm{m}$. Histograms represent the mean \pm S.E.M. $N=3$, ${ }^{*}<0.05, * \star P<0.01, * \star \star P<0.001$ via Student's t-tests and one-way ANOVA, Bonferroni's test was analyzed for post hoc comparisons. 


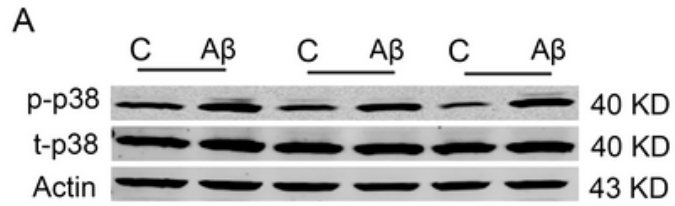

B
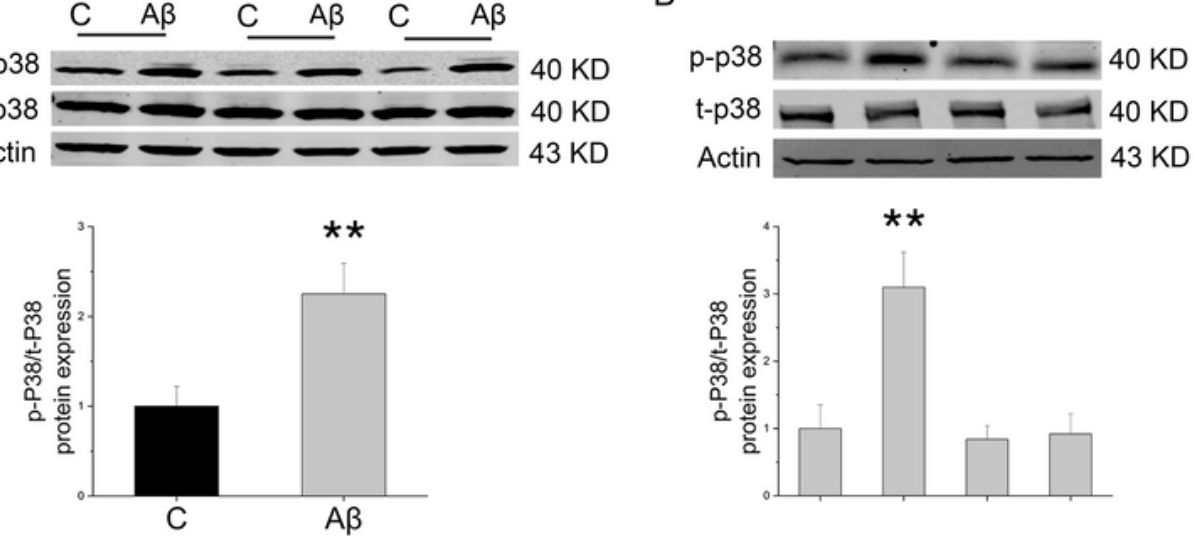

$\mathrm{AB}(2 \mu \mathrm{mol} / \mathrm{L})$ BIRB $796(10 \mu \mathrm{mol} / \mathrm{L})$

C

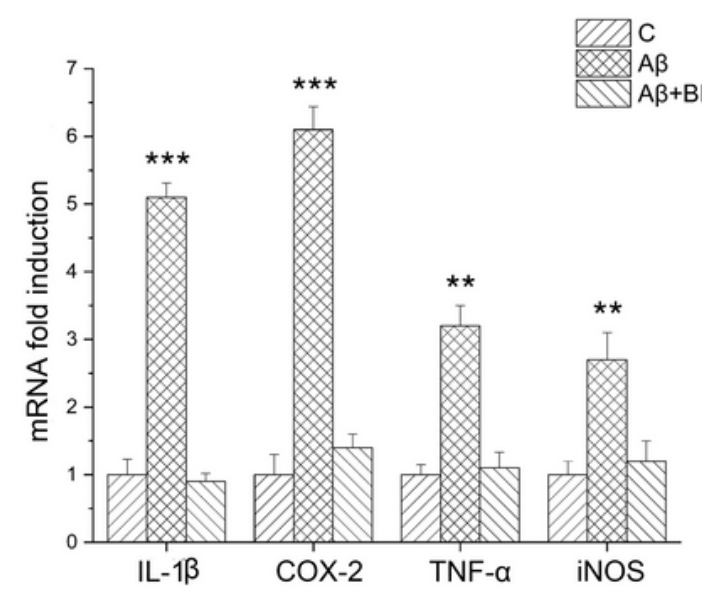

\section{Figure 5}

The expressions of p38 MAPK and its downstream inflammatory cytokines in A $31-42$ induced mice retinas and in the primary microglia with or without p38 inhibitor. (a) Western blot detection of p38 in mice retina on 3 days after subretinal injection of $A \beta 1-42$ or PBS. The primary microglial cells were

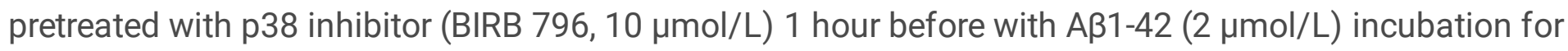
24 hours; the samples were then for (b) Western blot detection of the expression of p38 and (C) Q-PCR 
detection of IL-1 $\beta$, COX-2, TNF- $\alpha$ and iNOS mRNA expressions. p-p38, phosphorylated-p38; t-p38, totalp38. Histograms represent the mean \pm S.E.M. $N=3, * P<0.05$, $* \star P<0.01$, ${ }^{*} * \star P<0.001$ via Student's t-tests and one-way ANOVA, Bonferroni's test was analyzed for post hoc comparisons.

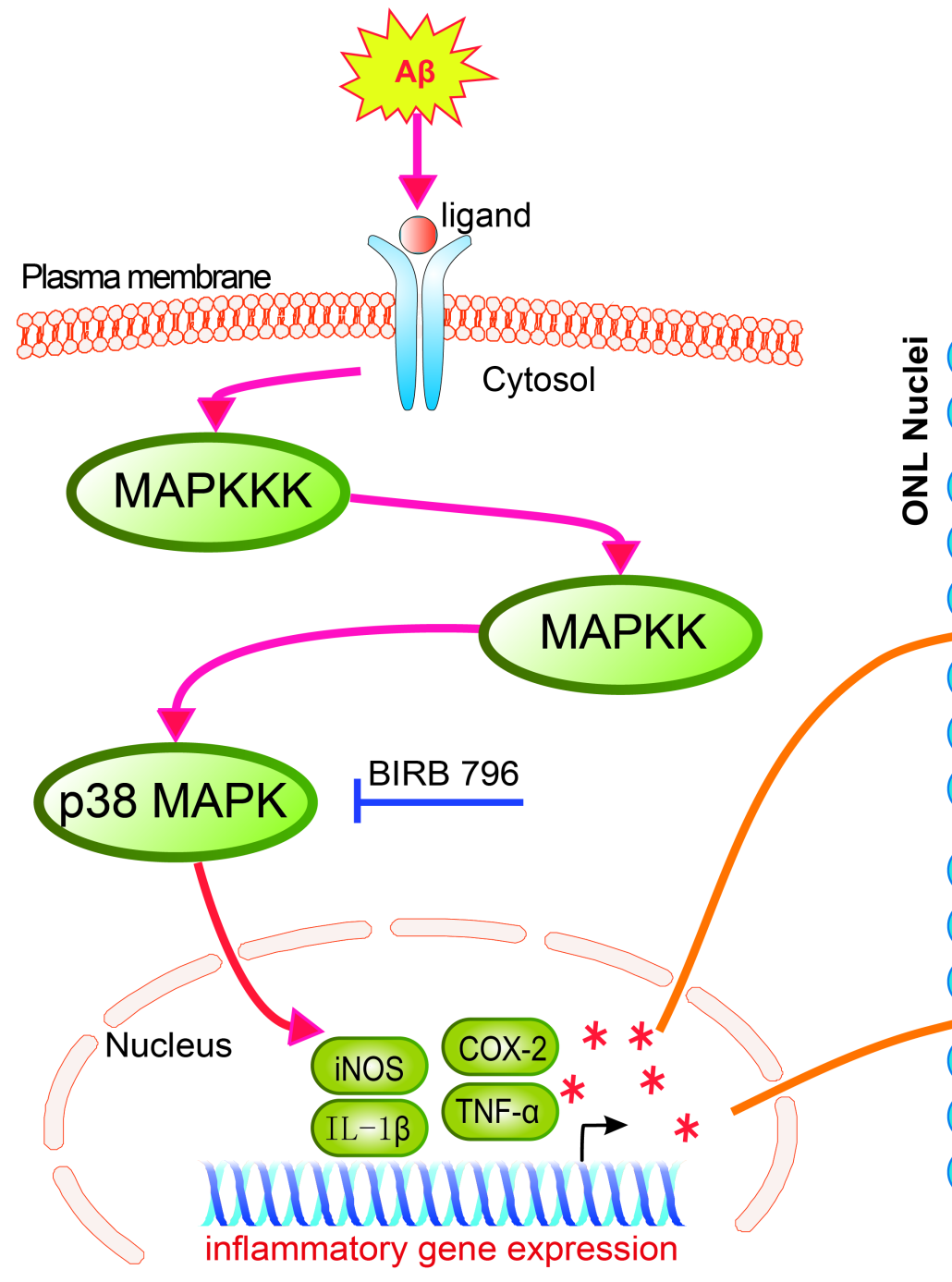

Microglia activation

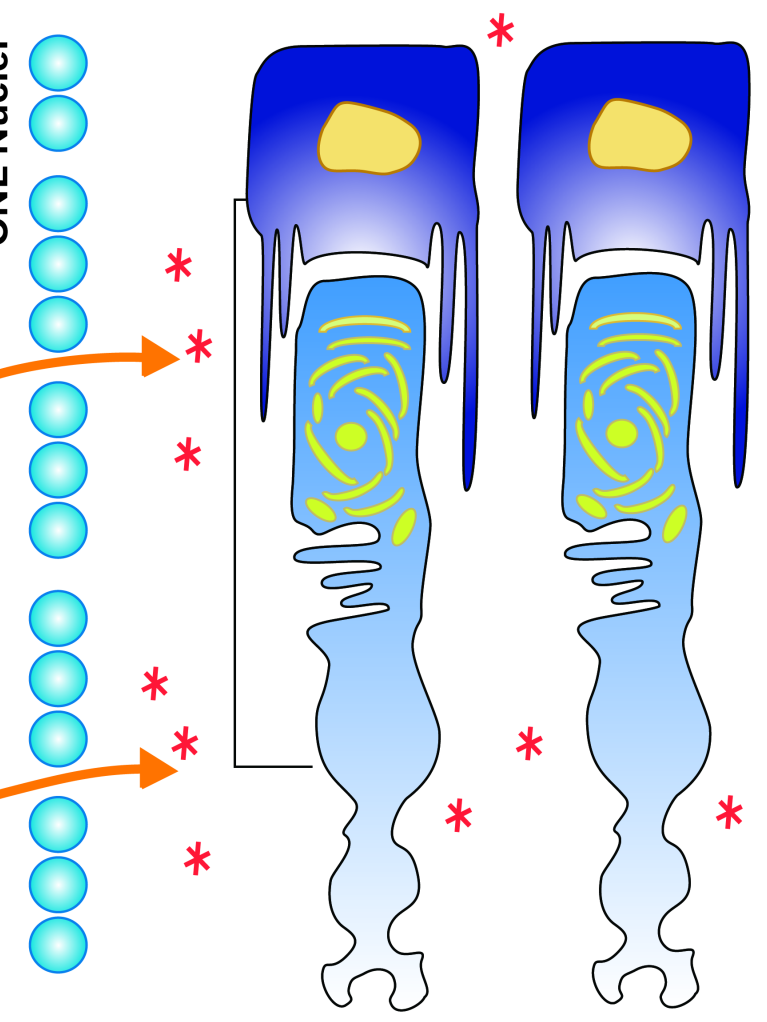

Photoreceptor apoptosis

* inflammatory cytokines

\section{Figure 6}

Schematic illustration of p38 MAPK signaling pathway and its downstream neuroinflammatory cytokines in activated microglia leading to photoreceptor apoptosis. 